Pacific Journal of Mathematics

ON THE SEARCH FOR WEIGHTED NORM INEQUALITIES

Nestor Edgardo Aguilera
AND ELEONor Ofelia Harbour De Aguilera 


\section{ON THE SEARCH FOR WEIGHTED NORM INEQUALITIES FOR THE FOURIER TRANSFORM}

\section{Néstor E. Aguilera and Eleonor O. Harboure}

B. Muckenhoupt posed in [1] the problem of characterizing those non-negative functions $u$ and $v$, which for some $p, 1 \leq p<\infty$, the inequality

$$
\int_{-\infty}^{+\infty}|\hat{f}(x)|^{p} u(x) d x \leq C \int_{-\infty}^{+\infty}|f(x)|^{p} v(x) d x
$$

holds for any $f$, where $\hat{f}$ denotes the Fourier transform of $f$. In this paper we deal only with the case where either $u \equiv 1$ or $v \equiv 1$, finding that when $v \equiv 1,1<p<2$, a necessary condition is that for any $r>0$,

$$
\left[\sum_{k=-\infty}^{+\infty}\left(\int_{r k}^{r(k+1)} u(x) d x\right)^{b}\right]^{1 / b} \leq C r^{p-1}
$$

where $b=2 /(2-p)$, and that a sufficient condition $(v \equiv 1,1 \leq p)$ is that for any measurable set $E$,

$$
\int_{E} u(x) d x \leq C|E|^{p-1} .
$$

Similar conditions are obtained for the case $u \equiv 1$. Although we will show that the sufficient condition is not necessary (in $\$ 4$, Corollary 1 and again in \$6, Corollary 3 and Remark 4), we were unable to obtain any conclusions on our necessary condition.

1. As far as we know, only sufficient conditions had been considered before, in the case where both $u$ and $v$ are powers of $|x|$ (see e.g. [2] for the trigonometric case), although our sufficient condition is somehow a restatement of a generalized Hausdorf-Young inequality (see e.g. [4, page 200]). In this connection we must point out the work of Hardy, Littlewood and Paley (see e.g. [5, Chapter VII, §8 and Chapter XII, §§3, 5 and 6]). By the way, both of our conditions may be easily translated to the trigonometric setting, where relationships between $f$ and its Fourier coefficients are considered, $f$ appearing either on the left or right hand side of the inequality. For a similar inequality to that of our necessary condition see, for instance, [5, Chapter XVI, Example 8, page 298] where, however, the exponents are less than 2.

In $\$ 2$ we give some introductory ideas which give some "feeling" for the subject and use these in $\$ 3$ to show a simple necessary condition when $v \equiv 1$ which is quite similar to the sufficient condition treated in $\$ 4$ where we also give several equivalent conditions. In $\$ 5$ we give some examples showing the gap between the conditions of the two previous sections so 
that the necessary condition of $\$ 6$ comes as no surprise. In this latter section we make several observations and give some examples, showing the implications between all three conditions. Finally, in $\$ 7$ we briefly consider the case $u \equiv 1$.

We will denote by $\hat{f}$ the Fourier transform of $f$ defined by

$$
\hat{f}(x)=\int_{-\infty}^{+\infty} e^{-i x y} f(y) d y .
$$

The corresponding inverse Fourier transform of $f$ will be denoted by $\check{f}$. $C$, as usual, stands for a constant which need not be the same at each occurrence, which may depend on $p$ but not on the general functions considered. We will work in just one dimension and omit limits of integration or summation when it is clear what they should be. Throughout the paper $u$ and $v$ will be non-negative measurable functions.

2. Preliminaries. When considering inequalities of the type

$$
\int|\hat{f}|^{p} u d x \leq C \int|f|^{p} v d x
$$

it is apparent that the particular behaviour of translations and dilations under the Fourier transform will be reflected on properties of $u$ and $v$. Let us recall that

(1) if $f_{\varepsilon}(x)=\frac{1}{\varepsilon} f(x / \varepsilon)$, then $\left(f_{\varepsilon}\right) \hat{(x)}=\hat{f}(\varepsilon x)$.

(2) if $g(x)=f(x+a)$, then $\hat{g}(x)=e^{i a x} \hat{f}(x)$ and the "reciprocal" to (2).

(3) if $g(x)=e^{i a x} f(x)$, then $\hat{g}(x)=\hat{g}(x-a)$.

Let us take for instance the case where $(*)$ holds and $u=v$ is locally integrable. Using (2) and (3) above and (*) twice it is not difficult to show that

$$
\int|f(-x+a)|^{p} u(x) d x \leq C \int|f(x+b)|^{p} u(x) d x
$$

for any choice of $a$ and $b$, since $\hat{f}(x)=2 \pi f(-x)$. If $f$ is the characteristic function of the interval $(-\varepsilon, \varepsilon)$ we may conclude that for any $\varepsilon>0$ and any $a$ and $b$,

$$
\frac{1}{2 \varepsilon} \int_{|x-a|<\varepsilon} u(x) d x \leq \frac{C}{2 \varepsilon} \int_{|x-b|<\varepsilon} u(x) d x .
$$

Since we are assuming that $u$ is locally integrable, for some $b=b_{0}$ we must have both

$$
M(b)=\sup _{0<\varepsilon<1} \frac{1}{2 \varepsilon} \int_{|x-b|<\varepsilon} u(x) d x<\infty
$$


and

$$
u(b)=\lim _{\varepsilon \rightarrow 0} \frac{1}{2 \varepsilon} \int_{|x-b|<\varepsilon} u(x) d x .
$$

Therefore for any $a$ we must have

$$
M(a)=\sup _{0<\varepsilon<1} \frac{1}{2 \varepsilon} \int_{|x-a|<\varepsilon} u(x) d x \leq C M\left(b_{0}\right)
$$

and for almost all $a$,

$$
u(a) \leq C u\left(b_{0}\right) .
$$

So we conclude that if $u$ is not identically zero (i.e. the set where $u$ is different from zero has positive measure), there exist non-zero constants $A$ and $B$ so that

$$
A \leq u(x) \leq B
$$

for almost all $x$. Hence, $u$ may be replaced by the funciton identically equal to 1 in $(*)$.

Now if $f(x)=e^{-x^{2} / 2}$, we know that $\hat{f}(x)=\sqrt{2 \pi} f(x)$, taking $f_{\varepsilon}(x)$ as in (1) above, we see that

$$
\int\left|\hat{f}_{\varepsilon}\right|^{p} d x=\frac{C_{1}}{\varepsilon}
$$

and

$$
\int\left|f_{\varepsilon}\right|^{p} d x=\frac{C_{2}}{\varepsilon^{p-1}}
$$

Applying (*) with $u \equiv v \equiv 1$, we must have for all $\varepsilon>0$,

$$
\frac{C_{1}}{\varepsilon} \leq C \frac{C_{2}}{\varepsilon^{p-1}}
$$

so that if $u \neq 0$, necessarily $p=2$.

This shows how different our weights must be from those considered when $\hat{f}$ is replaced in $(*)$ by the Hardy-Littlewood maximal function or the Hilbert transform (see e.g. [1]).

We will treat now the case $v \equiv 1$.

3. A simple necessary condition. Here we want to obtain properties on the non-negative function $u$ if the inequality

$$
\int|\hat{f}|^{p} u d x \leq C \int|f|^{p} d x
$$


holds, where $1 \leq p<\infty$. Recalling the behaviour of translations and dilations under the Fourier transform, we let

$$
f(x)=\chi_{(-1 / r, 1 / r)}(x) e^{i a x}
$$

where $\chi_{E}$ denotes the characteristic function of the set $E$.

Then

$$
f(x)=2 \frac{\sin ((x-a) / r)}{x-a}
$$

so that $|\hat{f}(x)| \geq 1 / r$ if $|x-a| \leq r$, and we must have, with $I=$ $\{x:|x-a| \leq r\}$,

$$
\int_{I} u(x) d x \leq r^{p} \int|\hat{f}|^{p} u d x \leq C r^{p} \int|f|^{p} d x=C r^{p-1}
$$

i.e. $u$ must be locally integrable and, denoting by $|E|$ the measure of the (measurable) set $E$,

$$
\int_{I} u(x) d x \leq C|I|^{p-1}
$$

for any interval $I$.

We observe that for $p=1$ this condition implies that $u$ is integrable over all of the real line and, by using Lebesgue's dominated convergence theorem, we see that $u$ must be bounded for $p=2$ and $u$ must vanish identically for $p>2$. So from now on we will restrict our attention to the case $1 \leq p \leq 2$.

4. A sufficient condition when $v \equiv 1,1 \leq p \leq 2$. A modification of the proof given in Zygmund's book [5, vol. 2, page 121] of a theorem of Paley will show that the following theorem holds:

THEOREM 1. Let $1 \leq p \leq 2$. If the locally integrable function $u$ satisfies the inequality

$$
\int_{E} u(x) d x \leq C|E|^{p-1}
$$

for any measurable set $E$, then

$$
\int|\hat{f}|^{p} u d x \leq C \int|f|^{p} d x
$$

Before giving the proof of Theorem 1, however we will give different equivalent conditions on $u$ : 
THEOREM 2. Let $1<p<2$ and let $b=2 /(2-p)$. Suppose $u$ is locally integrable, then the following are equivalent:

(i) $|\{x: u(x)>\lambda\}| \leq C / \lambda^{b / 2}$ for all $\lambda>0$.

(ii) $\int_{E} u d x \leq C|E|^{p-1}$ for all measurable sets $E$.

(iii) For $\alpha>b / 2$,

$$
\int_{\left\{x: u^{b / 2}(x) \leq \lambda\right\}} u^{\alpha}(x) d x \leq C_{\alpha} \lambda^{(2 \alpha / b)-1} \text { for all } \lambda>0 .
$$

(iv) For any $r>0, \lambda>0$,

$$
\#\left(\left\{k: \int_{k r}^{(k+1) r} u d x>\lambda\right\}\right) \leq C\left[\frac{r^{p-1}}{\lambda}\right]^{2 / b}
$$

where $\#(A)$ denotes the number of elements of the set $A$.

Proof of Theorem 2.

(i) implies (ii). Let $u^{*}(t)$ be defined for $t>0$ as the non-increasing rearrangement of $u$. Observe that since $\lambda^{-b / 2}$ is essentially the distribution function of $|x|^{p-2}$, for some constant $C>0$ we must have

$$
u^{*}(t) \leq C t^{p-2}
$$

and then

$$
\int_{E} u d x \leq \int_{0}^{|E|} u^{*} d x \leq C \int_{0}^{|E|} t^{p-2} d t=C|E|^{p-1}
$$

since $p>1$. (For the first inequality see for instance [5, vol 1, page 31].)

(ii) implies (i). Let $E=\{x: u(x)>\lambda\}, E_{n}=E \cap[-n, n]$. Then

$$
\left|E_{n}\right| \leq \frac{1}{\lambda} \int_{E_{n}} u d x \leq \frac{C}{\lambda}\left|E_{n}\right|^{p-1}
$$

so that

$$
\left|E_{n}\right| \leq \frac{C}{\lambda^{b / 2}}
$$

and letting $n$ go to infinity, we obtain

$$
|E| \leq \frac{C}{\lambda^{b / 2}}
$$


(i) implies (iii).

$$
\begin{aligned}
\int_{\left\{x: u^{b / 2}(x) \leq \lambda\right\}} u^{\alpha}(x) d x & \leq \sum_{n=0}^{\infty} \int_{\left\{x: 2^{-n-1} \lambda<u^{b / 2}(x) \leq 2^{-n} \lambda\right\}} u^{\alpha}(x) d x \\
& \leq C \sum_{n=0}^{\infty}\left(2^{-n} \lambda\right)^{2 \alpha / b} \cdot\left|\left\{x: u^{b / 2}(x)>2^{-n-1} \lambda\right\}\right| \\
& \leq C \sum_{n=0}^{\infty} \frac{\lambda^{2 \alpha / b-1}}{2^{n(2 \alpha / b-1)}}=C^{(2 \alpha / b)-1} .
\end{aligned}
$$

(iii) implies (i).

$$
\begin{aligned}
|\{x: u(x)>\lambda\}| & \leq \sum_{n=0}^{\infty}\left|\left\{x: 2^{n+1} \lambda \geq u(x)>2^{n} \lambda\right\}\right| \\
& \leq \sum_{n=0}^{\infty} \frac{1}{\left(2^{n} \lambda\right)^{b}} \int_{\left\{x: u(x) \leq 2^{n+1} \lambda\right\}} u^{\alpha}(x) d x \\
& \leq C \sum_{n=0}^{\infty} \frac{1}{\left(2^{n} \lambda\right)^{\alpha}}\left(2^{n} \lambda\right)^{-b / 2} \leq C \lambda^{-b / 2} .
\end{aligned}
$$

(i) implies (iv). Given $\lambda>0$ and $r>0$, set $\beta=(\lambda / 4 r)^{b / 2}$ and let $E=\left\{x: u^{b / 2}(x)>\beta\right\}$. Let $I_{k}$ denote the interval $(k r,(k+1) r)$ and assume $\int_{I_{k}} u d x>\lambda$. Then either $\int_{I_{k} \cap E} u d x>\lambda / 2$ or $\int_{I_{k} \cap e_{E}} u d x>\lambda / 2$, where $\mathcal{C E}$ is the complement of $E$. However, $\int_{I_{k} \cap e_{E}} u d x>\lambda / 2$ implies $\lambda / 2<\int_{I_{k} \cap e_{E}} u d x \leq \beta^{2 / b} r=\lambda / 4$, which is impossible. So

$$
\#\left(\left\{k: \int_{I_{k}} u d x>\lambda\right\}\right) \leq \#\left(\left\{k: \int_{I_{k} \cap E} u d x>\frac{\lambda}{2}\right\}\right) .
$$

Now

$$
\frac{\lambda}{2} \#\left(\left\{k: \int_{I_{k} \cap E} u d x>\frac{\lambda}{2}\right\}\right) \leq \sum_{k=-\infty}^{+\infty} \int_{I_{k} \cap E} u d x \leq \int_{E} u d x
$$

which, by (ii) and (i) is bounded by $C|E|^{p-1} \leq C \beta^{1-p}$. So

$$
\#\left(\left\{k: \int_{I_{k}} u d x>\lambda\right\}\right) \leq C\left(\frac{r}{\lambda}\right)^{(p-1) /(2-p)} \frac{1}{\lambda}=C\left(\frac{r^{p-1}}{\lambda}\right)^{2 / b} .
$$

(iv) implies (i). Let

$$
u_{n}(x)=2^{n} \int_{k / 2^{n}}^{(k+1) / 2^{n}} u(t) d t \quad \text { for } \frac{k}{2^{n}} \leq x<\frac{k+1}{2^{n}}
$$


Then $u_{n}$ converges to $u$ almost everywhere and hence in measure. Therefore

$$
|\{x: u(x)>\lambda\}| \leq \underline{\lim }\left|\left\{x: u_{n}(x)>\lambda / 2\right\}\right|
$$

but

$$
\begin{aligned}
\left|\left\{x: u_{n}(x)>\lambda\right\}\right| & =\frac{1}{2^{n}} \#\left(\left\{k: 2^{n} \int_{k / 2^{n}}^{(k+1) / 2^{n}} u(t) d t>\lambda\right\}\right) \\
& \leq \frac{C}{2^{n}}\left(\frac{\left(1 / 2^{n}\right)^{p-1}}{\lambda / 2^{n}}\right)^{1 /(2-p)}=\frac{C}{\lambda^{2 / p}} .
\end{aligned}
$$

We should observe that in Theorem 2 , if $p=1$ we have (i) implies (ii) but not the converse. Actually, in the proof of Theorem 1 we will only use condition (iii) when $1<p<2$. However it is important to put the condition in the form (i) which says that the distribution function of $u$ is bounded by that of $|x|^{2-p}$, showing the connection with the HausdorfYoung theorem and the Hardy, Littlewood and Paley results mentioned in the introduction. Condition (iii) is stated because of its remarkable similarity with the necessary condition found in $\S 3$. Condition (iv) will be useful for comparing the sufficient condition and the necessary one in $\S 6$.

We turn now to the proof of Theorem 1:

Proof of Theorem 1. As already mentioned, we will use Marcinkiewicz' interpolation theorem to interpolate between $p=1$ and $p=2$. Let $T$ be defined for $f \in L^{p} \cap L^{1}$ by

$$
T f(x)= \begin{cases}u^{-b / 2}(x) f(x) & \text { if } u(x) \neq 0 \\ 0 & \text { if } u(x)=0\end{cases}
$$

Then

$$
\int|\hat{f}|^{p} u d x=\int|T f|^{p} u^{b} d x
$$

where $b=2 /(2-p)$.

In $L^{1}$ we have the weak-type estimate

$$
\int_{\{x:|T f(x)|>\lambda\}} u^{b}(x) d x<\frac{C}{\lambda} \int|f| d x
$$

since $|\hat{f}(x)| \leq \int|f(t)| d t$ and (iii) of Theorem 2 is satisfied. On the other hand, in $L^{2}$, by Plancherel's identity we have the inequality

$$
\int|T f|^{2} u^{b} d x \leq C \int|f|^{2} d x .
$$


By interpolating, we obtain the result when $1<p<2$. If $p=1$, the condition $\int_{E} u \leq C$ for any measurable set $E$ implies $u \in L^{1}$, and since $|\hat{f}(x)| \leq \int|f(t)| d t$, the result is obvious. On the other hand, when $p=2$, $\int_{E} u d x \leq C|E|$ implies $|u(x)| \leq C$ and the result follows by Plancherel's identity.

It is interesting to observe that for $p=1$ or $p=2$ the simple necessary condition of $\$ 3$ and the sufficient condition of Theorem 1 are equivalent, in fact we used the former in the last proof when $p=1$ or $p=2$. However we cannot "interpolate" and obtain that they are equivalent for $1<p<2$, as we show in the following section.

5. Some examples. Let $p$ be strictly between 1 and 2 . Let $F$ be a measurable set such that $|F|<\infty$. It is not difficult to show that if we take $u=\chi_{F}$ then

$$
\int|\hat{f}|^{p} u d x=\int_{F}|\hat{f}|^{p} d x \leq C|F|^{2-p} \int|f|^{p} d x
$$

where $C$ may depend on $p$. (In fact it follows from the previous theorem, keeping track of the constants, or directly by using interpolation.) However, for some sets we can sharpen the inequality:

Let $E=\cup_{k=1}^{N}\left(2^{k}, 2^{k}+1\right)$, we will show that in this case the mapping $f \rightarrow \chi_{E} \hat{f}$ has norm essentially equivalent (depending on $p$ ) to $N^{(2-p) / 2 p}$. To prove this fact we will use the equivalence

$$
\int\left(\sum_{I \in \Delta}\left|S_{I} f\right|^{2}\right)^{p / 2} d x=\int|f|^{p} d x
$$

valid for $1<p<\infty$, where $\Delta$ stands for the collection of all dyadic intervals and $S_{I} f$ is defined by $\left(S_{I} f\right)=\chi_{I} \hat{f}$. (A precise statement and proof may be found in [3, page 104].)

Let $A_{k}=\left(2^{k}, 2^{k}+1\right)$ and let $I_{k}=\left(2^{k}, 2^{k+1}\right)$ so that $E=\cup_{1}^{N} A_{k}$ and $A_{k} \subset I_{k}$. Then

$$
\int_{E}|\hat{f}|^{p} d x=\left.\sum_{1}^{N} \int_{A_{k}}\left|\chi_{I_{k}} \hat{f}^{p} d x \leq C \sum_{1}^{N} \int\right| S_{I_{k}} f\right|^{p} d x
$$

where the last inequality follows by taking $A_{k}=F$ at the beginning of this paragraph. Using Hölder's inequality we derive

$$
\begin{aligned}
\int_{E}|\hat{f}|^{p} d x & \leq \int\left(\sum_{1}^{N}\left|S_{I_{k}} f\right|^{2}\right)^{p / 2} N^{(2-p) / 2} d x \\
& \leq C N^{(2-p) / 2} \int|f|^{p} d x .
\end{aligned}
$$


To see that indeed the norm is not much smaller than $N^{(2-p) / 2 p}$, we take $f$ so that $\hat{f}=\chi_{E}$. Then

$$
\int_{E}|\hat{f}|^{p} d x=\int_{E} d x=N
$$

and

$$
\int|f|^{p} d x \simeq \int\left(\sum_{1}^{N}\left|S_{I_{k}} f\right|^{2}\right)^{p / 2} d x
$$

but

$$
\left|S_{I_{k}} f\right|=\left|\check{\chi}_{I}\right|, \text { where } I=(0,1) \text {. }
$$

Therefore

$$
\int|f|^{p} d x \simeq N^{p / 2}
$$

and so

$$
\int_{E}|\hat{f}|^{p} d x \simeq N^{1-p / 2} \int|f|^{p} d x
$$

As an application of this example we have the following.

COROLlaRY 1. The condition $\int_{E} u(x) d x \leq C|E|^{p-1}$ for any measurable set $E$ is not necessary for (**) to hold if $1<p<2$.

Proof. Let $E(\varepsilon, N)=\cup_{k=1}^{N}\left(\varepsilon 2^{k}, \varepsilon\left(2^{k}+1\right)\right)$. By using the behaviour of dilations under the Fourier transform and the previous example we get

$$
\int_{E(\varepsilon, N)}|\hat{f}|^{p} d x \leq\left(\varepsilon^{2} N\right)^{1 / b} \int|f|^{p} d x
$$

Let now $\varepsilon_{n}=2^{-n}, N_{n}=2^{n}$,

$$
E_{n}=2^{N_{n}+1}+E\left(\varepsilon_{n}, N_{n}\right)=\bigcup_{k=1}^{2^{n}}\left(2^{2^{n}+1}+2^{k-n}, 2^{2^{n}+1}+\left(2^{k}+1\right) 2^{-n}\right) .
$$

Finally let $u=\sum_{n=1}^{\infty} \chi_{E_{n}}$. Since the $E_{n}$ 's are disjoint, we have

$$
\int|\hat{f}|^{p} u d x=\sum_{n=1}^{\infty} \int_{E_{n}}|\hat{f}|^{p} d x \leq C \sum_{n=1}^{\infty} \frac{1}{2^{n / b}} \int|f|^{p} d x \leq C \int|f|^{p} d x .
$$

So $(* *)$ holds. On the other hand, for $\lambda<1$,

$$
|\{x: u(x)>\lambda\}|=\sum_{n}\left|E_{n}\right|=\infty
$$

and condition (i) of Theorem 2 is not satisfied. 
Corollary 2. The condition $\int_{I} u d x \leq C|I|^{p-1}$ for any interval $I$ is not sufficient for $(* *)$ to hold if $1<p<2$.

Proof. Take $u=\chi_{E}$, where $E=\cup_{k=1}^{\infty}\left(2^{k}, 2^{k}+1\right)$. It is easy to see that $(* *)$ does not hold for $u$, because of the example given at the beginning of this section letting $N \rightarrow \infty$. On the other hand, the condition of $\S 3$ is satisfied since

$$
2^{j}<|I| \leq 2^{j+1} \quad \text { for } j>0,
$$

implies

$$
\int_{I} u d x \leq \int_{0}^{2^{j+1}} u d x=j \leq C \log |I| .
$$

REMARK 1. It is a curious fact that in both corollaries, the function $u$ that was found is independent of $p$.

6. A stronger necessary condition. The preceding paragraph shows that we must get an intermediate condition for $1<p<2$. It is possible to obtain the following

TheOrem 3. If $1<p<2$ and $u$ satisfies

$$
\int|\hat{f}|^{p} u d x \leq C \int|f|^{p} d x
$$

then $u$ must satisfy the inequality

$$
\left(\sum_{k=-\infty}^{+\infty}\left(\int_{k r}^{(k+1) r} u(x) d x\right)^{b}\right)^{1 / b} \leq C r^{p-1}
$$

for any $r>0$, where $b=2 /(2-p)$.

Proof. Let $g(x)=\Sigma_{k} a_{k} \chi_{(k, k+1)}$, where $a_{k}=0$ except for finitely many $k$ 's. Let $f$ be defined by $f(x)=g(x)$, i.e.

$$
f(x)=\sum_{k} a_{k} e^{i k x} \frac{\left(e^{i x}-1\right)}{2 \pi i x} .
$$

Let us estimate firstly the $L^{p}$-norm of $f$.

$$
\int|f|^{p} d x=\int_{-\pi}^{\pi}|f(x)|^{p} d x+\sum_{n \neq 0} \int_{-\pi+n 2 \pi}^{\pi+n 2 \pi}|f(x)|^{p} d x .
$$

For $n \neq 0$,

$$
\int_{-\pi+n 2 \pi}^{\pi+n 2 \pi}|f(x)|^{p} d x \leq \frac{C}{|n|^{p}} \int_{-\pi}^{\pi} \mid \sum a_{k} e^{\left.i k x\right|^{p}} d x
$$


and also

$$
\int_{-\pi}^{\pi}|f(x)|^{p} d x=\int_{-\pi}^{\pi}\left|\sum a_{k} e^{i k x}\right|^{p} d x
$$

since $\left|\left(e^{i x}-1\right) / x\right|$ remains bounded by above and by below away from zero if $|x|<\pi$. Therefore

$$
\int_{-\infty}^{+\infty}|f|^{p} d x \cong \int_{-\pi}^{\pi}\left|\sum a_{k} e^{i k x}\right|^{p} d x
$$

On the other hand,

$$
\left.\int\left|\hat{f}^{p} u d x=\sum_{k}\right| a_{k}\right|^{p} \int_{k}^{k+1} u(x) d x .
$$

Since we must have

$$
\sum_{k}\left|a_{k}\right|^{p} \int_{k}^{k+1} u(x) d x \leq C \int_{-\pi}^{\pi}\left|\sum a_{k} e^{i k x}\right|^{p} d x \leq C\left(\sum_{k}\left|a_{k}\right|^{2}\right)^{p / 2}
$$

for any choice of the (finite) sequence $a_{k}$, we obtain our result with $r=1$.

The case of general $r>0$ is obtained from the previous one by considering dilations: if $f_{\varepsilon}(x)=\frac{1}{\varepsilon} f(x / \varepsilon)$, then $\left(f_{\varepsilon}\right) \hat{(}(x)=\hat{f}(\varepsilon x)$ and $\int\left|f_{\varepsilon}\right|^{p} d x=\left(\varepsilon^{p-1}\right)^{-1} \int|f|^{p} d x$, choosing $\varepsilon=\frac{1}{r}$, the result follows.

Several remarks are in order:

REMARK 2. It should be clear that this new condition stays in between the earlier two: it implies that of $\S 3$ obviously and is strictly stronger by the example in Corollary 2 in $\$ 5$. It is implied by the sufficient condition of course, but it is weaker by Corollary 1 of $\$ 5$. This may be also seen by (iv) of Theorem 2, a result which we state as a corollary.

Corollary 3. Let $u$ be locally integrable and let $1<p<2$. Suppose for any measurable $E$,

$$
\int_{E} u d x \leq C|E|^{p-1}
$$

then for any $\alpha>1 /(2-p)=b / 2$ and any $r>0$

$$
\left(\sum_{k=-\infty}^{+\infty}\left(\int_{k r}^{k(r+1)} u d x\right)^{\alpha}\right)^{1 / \alpha} \leq C r^{p-1}
$$

Proof. We just observe that for $\alpha=b / 2$, we have a weak-type inequality by (iv) in Theorem 2 of $\S 4$.

REMARK 3. The necessary condition in Theorem 3 cannot be changed much as for the lengths of the intervals: we cannot consider arbitrary 
lengths in the same sum. For suppose $u(x)=|x|^{p-2}$, for which (**) holds, and let $I_{k}=\left(2^{k}, 2^{k+1}\right)$, then

$$
\sum_{k=-\infty}^{+\infty}\left(\frac{1}{\left|I_{k}\right|^{p-1}} \int_{I_{k}} u d x\right)^{\alpha}=\infty
$$

for any $\alpha$.

Remark 4. The exponent $b=2 /(2-p)$ cannot be lowered. For let $\left\{\gamma_{k}\right\}_{k=1}^{\infty}$ be a sequence of positive numbers such that $\sum_{k=1}^{\infty} \gamma_{k}^{b}<\infty$, and let $u=\sum_{k=1}^{\infty} \gamma_{k} \chi_{\left(2^{k}, 2^{k}+1\right)}$, then $(* *)$ holds. For let $f \in L^{p}$,

$$
\int|\hat{f}|^{p} u d x=\sum_{k=1}^{\infty} \gamma_{k} \int_{2^{k}}^{2^{k}+1}|\hat{f}|^{p} d x=\sum_{k=1}^{\infty} \gamma_{k} \int_{2^{k}}^{2^{k}+1}\left|\left(S_{k} f\right)^{n}\right|^{p} d x
$$

where $S_{k} f$ is defined by $\left(S_{k} f\right) \hat{f} \hat{f} \cdot \chi_{\left(2^{k}, 2^{k}+1\right)}$. By what was said at the beginning of $\S 5$ and the result on dyadic decomposition mentioned there, we have

$$
\begin{aligned}
\int|\hat{f}|^{p} u d x & \leq C \sum_{k=1}^{\infty} \gamma_{k} \int_{-\infty}^{+\infty}\left|S_{k} f\right|^{p} d x=C \int_{-\infty}^{+\infty}\left(\sum_{k=1}^{\infty} \gamma_{k}\left|S_{k} f\right|^{p}\right) d x \\
& \leq C \int_{-\infty}^{+\infty}\left(\sum_{k=1}^{\infty} \gamma_{k}^{b}\right)^{1 / b}\left(\sum_{k=-\infty}^{+\infty}\left|S_{k} f\right|^{2}\right)^{p / 2} d x \\
& \leq C\left(\sum_{k=1}^{\infty} \gamma_{k}^{b}\right)^{1 / b} \int_{-\infty}^{+\infty}|f|^{p} d x
\end{aligned}
$$

using Hölder's inequality to obtain the next to the last inequality. However, we may further ask $\sum_{k=1}^{\infty} \gamma_{k}^{\alpha}=\infty$ for $\alpha<b$, from where

$$
\sum_{j=-\infty}^{+\infty}\left(\int_{j}^{j+1} u d x\right)^{\alpha}=\infty
$$

for any such $\alpha$.

7. The case $u \equiv 1$. Here we want to examine those $v$ 's for which, for some $p, 1<p<\infty$,

$$
\int|\hat{f}|^{p} d x \leq C \int|f|^{p} v d x
$$

An immediate consequence of this inequality is the fact that the set where $v$ vanishes has measure zero, which follows by taking $f$ supported on that set. There is a duality between this case and the one treated in the previous section. For let $q$ be the dual expoent $p, 1 / p+1 / q=1$, and let 
$u(x)=v^{-q / p}(x)=v^{1 /(p-1)}(x)(u(x)=0$ if $v(x)=\infty)$. Then $u$ satisfies an inequality of the type $(* *)$, since

$$
\int|f|^{q} u d x=\int\left|\hat{f} u^{1 / q}\right|^{q} d x=\sup _{g \in \Delta} \int \hat{f} g d x
$$

where $\Delta=\left\{g: \int|g|^{p} v d x \leq 1\right\}$. (To see this we have to use the property of the set of zeros of $v$.) We have, if $g \in \Delta$,

$$
\int \hat{f} g d x=\int f \hat{g} d x \leq C\left(\int|f|^{q} d x\right)^{1 / q} .
$$

Conversely, if $u$ satisfies an inequality of the type

$$
\int|\hat{f}|^{q} u d x \leq \int|f|^{q} d x
$$

for some $q, 1<q<\infty$, then $u$ is locally integrable and the set where $u$ is infinite has measure zero. If $1 / p+1 / q=1$, we define $v(x)=u^{-p / q}(x)$ if $u(x) \neq 0$ and $v(x)=\infty$ if $u(x)=0$. A similar reasoning shows then that $(* * *)$ holds for this $v$.

We thus obtain several properties, similar to those obtained in the previous section, whose proofs are obtained either by duality or by using a reasoning analogous to those of section II, so we will state them without proof.

PROPOSITION 1. If $(* * *)$ holds then v satisfies

$$
\int_{I} v(x) d x \geq C|I|^{p-1}
$$

for any interval I. In particular for $p<2$ only the trivial case $v \equiv \infty$ (in the almost everywhere sense) is admissible. For $p=2$, this condition implies that $v(x) \geq C>0$ almost everywhere, which is also a sufficient condition.

Proposition 2. Suppose $2<p<\infty$ and $v$ satisfies any of the following equivalent conditions

(a) $\int_{E} v(x) d x \geq C|E|^{p-1}$ for any measurable set $E$

(b) $|\{x: v(x) \leq \delta\}| \leq C \delta^{1 / p-2}$ for any $\delta>0$

(c) $\int_{\left\{x: v(x) \geq \lambda^{p-2}\right\}} v^{2 /(p-2)}(x) d x \leq C / \lambda$ for any $\lambda>0$ then $(* * *)$ holds.

Proposition 3. If $(* * *)$ holds, then for any $r>0$,

$$
\left(\sum_{k=-\infty}^{+\infty}\left(\int_{r k}^{r(k+1)} v^{-1 /(p-1)}(x) d x\right)^{\alpha}\right)^{1 / \alpha} \leq C r^{1 /(p-1)}
$$

where $\alpha=2(p-1) /(p-2)$. 


\section{REFERENCES}

1. B. Muckenhoupt, Weighted norm inequalities for classical operators, Proc. Symp. in Pure Math., 34, part 1 (1979), 69-83.

2. E. M. Stein, Interpolation of linear operators, Trans. Amer. Math. Soc., 83 (1956), $482-492$.

3. Singular Integrals and Differentiability Properties of Functions, Princeton University Press, Princeton (1970).

4. E. M. Stein and G. Weiss, Introduction to Fourier Analysis on Euclidean Spaces, Princeton University Press, Princeton (1975).

5. A. Zygmund, Trigonometrical Series, 2nd edition, 2 vols., Cambridge University Press, Cambridge, Eng. (1968).

Received September 24, 1980. Miembro de la Carrera del Investigador del CONICET. INTEC-PEMA-Programa Especial de Matemática Aplicada Santa Fe en el Instituto de Desarrollo Tecnológico para la Industria Química, dependiente del Consejo Nacional de Investigaciones Cientificas y Técnicas (CONICET).

InSTITUTo DE Desarrollo TeCnológico

PARA LA INDUSTRIA QUIMICA

SANTIAGo DEL ESTERO 2654

3000 Santa Fe, Argentina 


\section{PACIFIC JOURNAL OF MATHEMATICS \\ EDITORS}

DONALD BABBITT (Managing Editor)

University of California

Los Angeles, CA 90024

\section{Hugo Rossi}

University of Utah

Salt Lake City, UT 84112

C. C. Moore and Arthur Ogus

University of California

Berkeley, CA 94720
J. DugundiI

Department of Mathematics

University of Southern California

Los Angeles, CA 90089-1113

R. FINN and H. SAMELSON

Stanford University

Stanford, CA 94305

ASSOCIATE EDITORS
R. ARens
E. F. BECKENBACH
B. H. NeumanN
F. WOLF
K. YoshidA (1906-1982)

\section{SUPPORTING INSTITUTIONS}

UNIVERSITY OF ARIZONA

UNIVERSITY OF BRITISH COLUMBIA

CALIFORNIA INSTITUTE OF TECHNOLOGY

UNIVERSITY OF CALIFORNIA

MONTANA STATE UNIVERSITY

UNIVERSITY OF NEVADA, RENO

NEW MEXICO STATE UNIVERSITY

OREGON STATE UNIVERSITY
UNIVERSITY OF OREGON

UNIVERSITY OF SOUTHERN CALIFORNIA

STANFORD UNIVERSITY

UNIVERSITY OF HAWAII

UNIVERSITY OF TOKYO

UNIVERSITY OF UTAH

WASHINGTON STATE UNIVERSITY

UNIVERSITY OF WASHINGTON 


\section{Pacific Journal of Mathematics}

Vol. 104, No. 1

May, 1983

Nestor Edgardo Aguilera and Eleonor Ofelia Harboure de Aguilera, On

the search for weighted norm inequalities for the Fourier transform $\ldots \ldots .1$

Jin Akiyama, Frank Harary and Phillip Arthur Ostrand, A graph and its complement with specified properties. VI. Chromatic and achromatic numbers ......................................... 15

Bing Ren Li, The perturbation theory for linear operators of discrete type . . . 29

Peter Botta, Stephen J. Pierce and William E. Watkins, Linear

transformations that preserve the nilpotent matrices .............. 39

Frederick Ronald Cohen, Ralph Cohen, Nicholas J. Kuhn and Joseph

Alvin Neisendorfer, Bundles over configuration spaces .......... 47

Luther Bush Fuller, Trees and proto-metrizable spaces . . . . . . . . . . 55

Giovanni P. Galdi and Salvatore Rionero, On the best conditions on the

gradient of pressure for uniqueness of viscous flows in the whole space . . 77

John R. Graef, Limit circle type results for sublinear equations $\ldots \ldots \ldots \ldots 85$

Andrzej Granas, Ronald Bernard Guenther and John Walter Lee,

Topological transversality. II. Applications to the Neumann problem for

$y^{\prime \prime}=f\left(t, y, y^{\prime}\right) \ldots \ldots \ldots \ldots \ldots \ldots \ldots \ldots \ldots \ldots \ldots \ldots \ldots \ldots \ldots . \ldots 5$

Richard Howard Hudson and Kenneth S. Williams, Extensions of

theorems of Cunningham-Aigner and Hasse-Evans . . . . . . . . . . . 111

John Francis Kurtzke, Jr., Centralizers of irregular elements in reductive algebraic groups

James F. Lawrence, Lopsided sets and orthant-intersection by convex

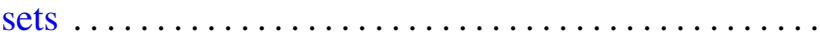

Åsvald Lima, G. H. Olsen and U. Uttersrud, Intersections of $M$-ideals and

$G$-spaces

Wallace Smith Martindale, III and C. Robert Miers, On the iterates of derivations of prime rings

Thomas H. Pate, Jr, A characterization of a Neuberger type iteration procedure that leads to solutions of classical boundary value problems

Carl L. Prather and Ken Shaw, Zeros of successive iterates of multiplier-sequence operators

Billy E. Rhoades, The fine spectra for weighted mean operators

Rudolf J. Taschner, A general version of van der Corput's difference theorem

Johannes A. Van Casteren, Operators similar to unitary or selfadjoint ones 